VOL. 44 (1991) [1-9].

\title{
ON METRIC REGULARITY OF MULTIFUNCTIONS
}

\section{A. Jourani}

The aim of this paper is to give a metric regularity theorem for multifunctions between metric spaces involving some known results for multifunctions by using the notion of strict $(G, \delta)$-differentiability of multifunctions and a simple convergence procedure.

\section{INTRODUCTION}

The notion of metric regularity of multifunctions (see Definition 1.1) plays an important role in optimisation theory, in particular in mathematical programming problems (see for example $[5,8,15,19,23,26,27,28]$ ). This concept has been extensively studied in varying degrees of generality by many authors (see $[3,5,6,14,16,17,18$, 21, 22, 24]). Probably the first result goes back to Graves [14], stating that a continuously differentiable mapping $F$ between Banach spaces whose derivative $D F\left(x_{0}\right)$ at $x_{0}$ is surjective is metrically regular around $\left(x_{0}, F\left(x_{0}\right)\right)$. Extending the well-known Banach perturbation lemma [20, Theorem 3 (2.V)], Robinson [24] has made explicit the metric regularity result for convex multifunctions in Banach spaces. Applying Ekeland's variational principle [11], Ioffe [16] and Borwein [5] proved some metric regularity results for Lipschitz mappings between Banach spaces. We can also consider that the results obtained by the authors $[1,2,4,7,13,25]$ are metric regularity results since one can establish a relationship between the metric regularity and the pseudo-Lipschitzianity of multifunctions (see [22] and [6]). Robinson [24] established that a multifunction $F$ between Banach spaces with convex closed graph which is open at a point $\left(x_{0}, y_{0}\right)$ of its graph is regular around $\left(x_{0}, y_{0}\right)$ and conversely. Without convexity of its graph the openness of $F$ at $\left(x_{0}, y_{0}\right)$ is not sufficient to recover the metric regularity of $F$ around $\left(x_{0}, y_{0}\right)$ (see Example 4.6 in [6]). Recently Penot [22] and Borwein and Zhuang [6] showed that the openness of $F$ around $\left(x_{0}, y_{0}\right)$ is equivalent to the metric regularity of $F$ around $\left(x_{0}, y_{0}\right)$.

In this paper we study metric regularity properties of multifunctions in metric spaces. Namely, we show that a multifunction $F$ preserves some properties of its derivative, in particular the openness and the metric regularity properties.

Received 28 June 1990

The author is indebted to Professors $\mathrm{L}$. Thibault and $\mathrm{D}$. Azé for valuable remarks helping to improve the paper.

Copyright Clearance Centre, Inc. Serial-fee code: 0004-9729/91 \$A2.00+0.00. 
To do this we introduce the notion of strict $(G, \delta)$-differentiability of multifunctions. To this end, let us introduce some notation.

Let $X, Y$ and $Z$ be linear spaces equipped with some distance $d$ which is invariant by translations, that is, $d(b+a, c+a)=d(b, c)$; let $F: X \rightrightarrows Y, L: X \rightrightarrows Y$ and $G: Z \rightrightarrows X$ be multifunctions and let $\delta: \mathbb{R}_{+} \rightarrow \mathbb{R}_{+}$be a strictly monotone continuous function. We denote by

$$
\operatorname{Gr} F=\{(x, y) \in X \times Y: y \in F(x)\}
$$

the graph of $F$ and by $B_{X}, B_{Y}$ and $B_{Z}$ the closed unit balls of $X, Y$ and $Z$ respectively. We denote by $F^{-1}$ the multifunction whose graph is deduced from $G r F$ by exchanging $x$ and $y$ and we set

$$
L \circ G(z)=\{y \in Y: \exists x \in G(z) ; y \in L(x)\} .
$$

Observe that, for each $y \in Y$,

$$
(L \circ G)^{-1}(y)=G^{-1} \circ L^{-1}(y)=\{z \in Z: y \in L \circ G(z)\} .
$$

Definition 0.1: $F$ is said to be strictly $(G, \delta)$-differentiable at $\left(x_{0}, y_{0}\right) \in G r F$ if there exists a multifunction $L: X \rightrightarrows Y$ such that for each $\varepsilon>0$, there exists $r>0$ such that

$$
L o G(z) \cap B_{Y} \subset F(x+G(z))-y+\varepsilon \delta(d(0, z)) B_{Y}
$$

for all $x \in x_{0}+r B_{X}, y \in\left(y_{0}+r B_{Y}\right) \cap F(x)$ and $z \in r B_{Z}$.

REMARK. When $X=Z, G(x)=x$ and $\delta(t)=t$, we recover the notion of strict differentiability of a multifunction (see [4] and [9]).

\section{The Main Result}

Definition 1.1: A multifunction $F$ is said to be metrically $\delta$-regular around $\left(x_{0}, y_{0}\right) \in G r F$ if there exist $K>0$ and $r>0$ such that

$$
d\left(x, F^{-1}(y)\right) \leqslant K \delta^{-1}(d(y, F(x)))
$$

for all $x \in x_{0}+r B_{X}$ and $y \in y_{0}+r B_{Y}$ where

$$
d(v, D)=\inf \left\{d\left(v, v^{\prime}\right): v^{\prime} \in D\right\} .
$$

Definition 1.2: $F$ is nicely $\delta$-invertible around $y_{0} \in F\left(x_{0}\right)$ if there exist $\alpha>0$ and $a>0$ such that for all $y \in y_{0}+\alpha B_{Y}$ there exists $x \in F^{-1}(y)$ such that $\delta\left(d\left(x_{0}, x\right)\right) \leqslant a d\left(y_{0}, y\right)$. 
REMARK. If $F$ is $\delta$-regular around $\left(x_{0}, y_{0}\right) \in G r F$ and if $\delta$ is continuous and strictly monotone then $F$ is nicely $\delta$-inversible around $y_{0} \in F\left(x_{0}\right)$.

Let us assume that $G$ and $\delta$ satisfy the following:

$H(G)$ : There exist $\alpha>0$ and $b>0$ such that

$$
G(z) \subset b d(0, z) B_{X} \text { for each } z \in \alpha B_{Z} .
$$

$H(\delta): \quad \delta(1)=1$ and for all $t, s \in[0,+\infty)$

$$
\delta^{-1}(t s) \leqslant \delta^{-1}(t) \delta^{-1}(s) .
$$

EXAmple. $Z=\mathbb{R}_{+}, G(t)=t^{*} B_{X}$ and $\delta(t)=t^{r}$ with $s \geqslant 1$ and $r>0$.

Theorem 1.3. Let $L$ be a strict $(G, \delta)$-derivative of $F$ at $\left(x_{0}, y_{0}\right) \in G r F$. Suppose that:

(i) $X$ is complete and $F$ has a closed graph.

(ii) $L \circ G$ is nicely $\delta$-invertible around $0 \in(L \circ G)(0)$.

(iii) $G$ and $\delta$ satisfy respectively $H(G)$ and $H(\delta)$.

Then $F$ is $\delta$-regular around $\left(x_{0}, y_{0}\right)$. More precisely there exist $a>0$ and $b>0$ such that for some $\varepsilon>0$ there is $s>0$ with:

$$
d\left(x, F^{-1}(y)\right) \leqslant \frac{b \delta^{-1}(a)}{1-\delta^{-1}(\varepsilon a)} \delta^{-1}(d(y, F(x)))
$$

for all $x \in x_{0}+s B_{X}$ and $y \in y_{0}+s B_{Y}$.

Proof: We follow the lines of Azé [4]. Since $L \circ G$ is nicely $\delta$-invertible there exist $a>0$ and $\alpha>0$ (we can choose $\alpha$ as in $H(G)$ ) such that for all $y \in \alpha B_{Y}$ we can exhibit $z \in G^{-1} \circ L^{-1}(y)$ satisfying:

$$
\delta(d(0, z)) \leqslant \operatorname{ad}(0, y) .
$$

From the strict $(G, \delta)$-differentiability of $F$ at $\left(x_{0}, y_{0}\right)$ it follows that for all $\varepsilon>0$ (with $\varepsilon a<1$ ) there exists $r>0$ such that

$$
L \circ G(z) \cap B_{Y} \subset F(x+G(z))-y+\varepsilon \delta(d(0, z)) B_{Y}
$$

for all $x \in x_{0}+r B_{X}, y \in\left(y_{0}+r B_{Y}\right) \cap F(x)$ and $z \in r B_{Z}$. Choose $\eta>0$ such that $\max (\eta,(\delta(\eta)) / a) \leqslant \min \left(\alpha, r, r\left(1-\delta^{-1}(\varepsilon a)\right) /\left(b \delta^{-1}(\varepsilon a)\right)\right)$ where $b$ is given by $H(G)$. Let $y \in y_{0}+(\delta(\eta) / a) B_{y}$. We shall construct a sequence $\left(\left(x_{n}, y_{n}\right)\right) \subset G r F$ such that $\left(\left(x_{n}, y_{n}\right)\right)$ converges to $(x, y)$ with $y \in F(x)$ and $x \in\left(x_{0}+K \delta^{-1}\left(d\left(y, y_{0}\right)\right) B_{X}\right)$, 
where $K>0$ does not depend on $x$ and $y$. Put $x_{-1}=x_{0}$. Let $n \in \mathbb{N}$ be such that, for each $k \in[0, n]$,

$$
\begin{gathered}
x_{k} \in\left(x_{0}+r B_{X}\right), y_{k} \in F\left(x_{k}\right), d\left(y, y_{k}\right) \leqslant(\varepsilon a)^{k} d\left(y, y_{0}\right) \\
d\left(x_{k}, x_{k-1}\right) \leqslant b\left(\delta^{-1}(\varepsilon a)\right)^{k} \delta^{-1}\left(a d\left(y, y_{0}\right)\right)
\end{gathered}
$$

We want to show that (1.3.3) holds for $k=n+1$. Since $y-y_{n} \in(\delta(\eta) / a) B_{Y}$, we derive from (1.3.1) the existence of $z_{n} \in G^{-1} \circ L^{-1}\left(y-y_{n}\right)$ such that $\delta\left(d\left(0, z_{n}\right)\right) \leqslant a d\left(y, y_{n}\right)$. It follows from (1.3.2) that there exist $u_{n} \in G\left(z_{n}\right)$ and $y_{n+1} \in F\left(x_{n}+u_{n}\right)$ such that

$$
\begin{aligned}
d\left(y, y_{n+1}\right) & \leqslant \varepsilon \delta\left(d\left(0, z_{n}\right)\right) \\
& \leqslant \varepsilon a d\left(y, y_{n}\right)
\end{aligned}
$$

Let us set $x_{n+1}=x_{n}+u_{n}$. Then, by assumption $H(G)$, we have

$$
\begin{aligned}
d\left(x_{n+1}, x_{n}\right) & =d\left(0, u_{n}\right) \leqslant b d\left(0, z_{n}\right) \\
& \leqslant b \delta^{-1}\left(a d\left(y, y_{n}\right)\right)
\end{aligned}
$$

On the other hand we obtain, by using the induction assumption (1.3.3), $H(G)$ and by the choice of $\eta$,

$$
\begin{aligned}
d\left(x_{n+1}, x_{0}\right) & \leqslant d\left(x_{n+1}, x_{n}\right)+\sum_{k=1}^{n} d\left(x_{k}, x_{k-1}\right) \\
& \leqslant \frac{b \delta^{-1}(a) \eta}{1-\delta^{-1}(\varepsilon a)} \\
& \leqslant r
\end{aligned}
$$

In virtue of (1.3.3), (1.3.4), (1.3.5) and (1.3.6) we obtain

$$
\begin{aligned}
& x_{n+1} \in\left(x_{0}+r B_{X}\right), y_{n+1} \in F\left(x_{n+1}\right) \\
& d\left(x_{n+1}, x_{n}\right) \leqslant b\left(\delta^{-1}(\varepsilon a)\right)^{n} \delta^{-1}\left(a d\left(y, y_{0}\right)\right) \\
& d\left(y_{n+1}, y\right) \leqslant(\varepsilon a)^{n+1} d\left(y, y_{0}\right)
\end{aligned}
$$

so that (1.3.3) holds for $k=n+1$. Observe that, for each $n, p \in \mathbb{N}$,

$$
\begin{aligned}
d\left(x_{n+p}, x_{n}\right) & \leqslant \sum_{k=1}^{p} d\left(x_{n+k}, x_{n+k-1}\right) \\
& \leqslant \frac{b}{1-\delta^{-1}(\varepsilon a)}\left(\delta^{-1}(\varepsilon a)\right)^{n}
\end{aligned}
$$


hence $\left(x_{n}\right)$ is a Cauchy sequence and then it converges to some $x \in X$. As $\left(y_{n}\right)$ converges to $y$, it ensues from the closedness of $\operatorname{Gr} F$ that $(x, y)$ belongs to $\operatorname{Gr} F$ which together with (1.3.6) yields

$$
y \in F(x) \text { and } x \in x_{0}+K \delta^{-1}\left(d\left(y, y_{0}\right)\right) B_{X}
$$

where $K=\left(b \delta^{-1}(a)\right) /\left(1-\delta^{-1}(\varepsilon a)\right)$. Now pick $y \in y_{0}+(\delta(\eta) / 2 a) B_{Y}$ and $x_{1} \in$ $x_{0}+(r / 2) B_{X}$ from (1.3.7); $F^{-1}(y) \neq \emptyset$ and $d\left(x_{1}, F^{-1}(y)\right)<+\infty$. Let us consider $y_{1} \in F\left(x_{1}\right) \cap\left(y_{0}+(\delta(\eta) / 2 a) B_{Y}\right)$; (if this set is empty, there is nothing to prove). The same argument as above applied to $\left(x_{1}, y_{1}\right)$ instead of $\left(x_{0}, y_{0}\right)$ provides the existence of $x \in x_{1}+r B_{X}$ such that $y \in F(x)$ and $x \in x_{1}+K \delta^{-1}\left(d\left(y, y_{1}\right)\right) \dot{B}_{X}$. Hence,

$$
d\left(x_{1}, x\right) \leqslant K \delta^{-1}\left(d\left(y, y_{1}\right)\right),
$$

and then,

$$
d\left(x_{1}, F^{-1}(y)\right) \leqslant K \delta^{-1}\left(d\left(y, F\left(x_{1}\right)\right) \cap\left(y_{0}+\frac{\delta(\eta)}{2 a} B_{Y}\right)\right)
$$

for all $x_{1} \in x_{0}+(r / 2) B_{X}$ and $y \in y_{0}+(\delta(\eta) / 2 a) B_{Y}$. As in Rockafellar [25] (see also Thibault [27]), one can show that there is $\gamma \in(0, r / 2)$ and $\beta \in(0, \delta(\eta) / 2 a)$ such that

$$
d\left(y, F\left(x_{1}\right) \cap\left(y_{0}+(\delta(\eta) / 2 a) B_{Y}\right)\right)=d\left(y, F\left(x_{1}\right)\right)
$$

for all $x_{1} \in x_{0}+\gamma B_{X}$ and $y \in y_{0}+\beta B_{Y}$, which completes the proof of the theorem. 0 REMARKs. (1) When $X=Z, G(x)=x$ and $\delta(t)=t$, we recover the results of [4] and [7].

(2) It follows from the proof of Theorem 1.3 that there is $b>0$ and $a>0$ such that for some $\varepsilon>0$ (with $\varepsilon a<1$ ) there is $s>0$ such that for all $y \in y_{0}+s B_{Y}$ there is $x \in F^{-1}(y)$ satisfying $d\left(x_{0}, x\right) \leqslant\left(b \delta^{-1}(a)\right)\left(\delta^{-1}\left(d\left(y_{0}, y\right)\right)\right) /\left(1-\delta^{-1}(\varepsilon a)\right)$.

The proof of the following theorem is exactly the same as that of Theorem 1.3.

THEOREM 1.4. Theorem 1.3 remains true if we replace the completeness of $X$ by the completeness of $\mathrm{GrF}$.

Corollary 1.5. If in Theorem 1.4 we assume that $L \circ G$ is $\delta$-regular around $(0,0) \in \operatorname{Gr}(L \circ G)$, then $F$ is $\delta$-regular around $\left(x_{0}, y_{0}\right)$.

Proof: It suffices to apply Theorem 1.4 since the $\delta$-regularity of $L \circ G$ implies the nice $\delta$-inversibility of $L \circ G$.

Borwein and Zhuang [6] have shown that, if for some strictly monotone continuous function $\delta^{\prime}, F$ is approximately $\delta^{\prime}$-open around $\left(x_{0}, y_{0}\right)$ (that is, there is $\alpha: \mathbb{R}_{+} \rightarrow \mathbb{R}_{+}$ 
with $\limsup _{t \downarrow 0}\left(\delta^{\prime-1}(\alpha(t))\right) /(t)<1$, and $r>0$ and $\eta>0$ such that for all $x \in x_{0}+r B_{X}$ and all $y \in F(x) \cap\left(y_{0}+\eta B_{Y}\right)$ and all $t \in(0, \eta)$,

$$
y+\delta^{\prime}(t) B_{Y} \subset \operatorname{cl}\left(F\left(x+t B_{X}\right)+\alpha(t) B_{Y}\right)
$$

where "cl" denotes the closure), then $F$ is $\delta$-regular around $\left(x_{0}, y_{0}\right)$ for all $\delta \leqslant \delta^{\prime}$ with $\limsup (\delta(t)) / t<+\infty$.

t10

In the following corollary we obtain the same result but with the condition $H\left(\delta^{\prime}\right)$ instead of $\limsup (\delta(t)) / t<+\infty$. Let us remark that from this corollary one can get information about the regularity constant $K$ of $F$. But first we give the following lemma:

Lemma 1.6. Let $c \in(0,1)$ and $\delta$ satisfy $H(\delta)$. Then for all $b \in[1 /(\delta(1 / c)), 1)$ and all $t>0$,

$$
\delta(c t) \leqslant b \delta(t)
$$

Proof: Consider the failure of (1.6). Then there is $b \in[1 /(\delta(1 / c)), 1)$ and $t>0$ such that $\delta(c t)>b \delta(t)$. This latter is equivalent to $1 / b>\delta(1 / c)$ thanks to $H(\delta)$ and the strict monotonicity of $\delta$ which contradicts $b \geqslant 1 /(\delta(1 / c))$.

Corollary 1.7 . Let $\delta$ satisfy $H(\delta)$. If $F$ is approximately $\delta$-open around $\left(x_{0}, y_{0}\right)$ and $G r F$ is complete then $F$ is $\delta$-regular around $\left(x_{0}, y_{0}\right)$.

Proof: The condition $\limsup _{t \downarrow 0}\left(\delta^{-1}(\alpha(t))\right) / t<1$ implies that there are $0<c<1$ and $\eta>0$ such that $\alpha(t)<\delta(c t)$ for all $t \in[0, \eta]$. Let $b \in[1 /(\delta(1 / c)), 1)$. Then by Lemma 1.6 and the approximately $\delta$-openness property of $F$ one has the existence of $r>0$ and $\gamma>0$ (we can assume that $\gamma=\eta$ ) such that.

$$
\begin{aligned}
\delta(t) B_{Y} & \subset F\left(x+t B_{X}\right)-y+(\delta(c t)-\alpha(t)+\alpha(t)) B_{Y} \\
& \subset F\left(x+t B_{X}\right)-y+\delta(c t) B_{Y} \\
& \subset F\left(x+t B_{X}\right)-y+b \delta(t) B_{Y}
\end{aligned}
$$

for all $x \in x_{0}+r B_{X}, y \in\left(y_{0}+\gamma B_{Y}\right) \cap F(x)$ and $t \in[0, \gamma]$. Let us set $\varepsilon=b,(\varepsilon<1)$, $Z=\mathbb{R}_{+}, G(t)=t B_{X}$ and $L(x)=\delta(d(0, x)) B_{Y}$. It is not difficult to see that the assumptions of Theorem 1.4 are satisfied.

REMARKs. (1) The result of Corollary 1.7 remains true for every strictly monotone continuous function $\delta^{\prime} \leqslant \delta$.

(2) It is not difficult to see that for some strictly monotone continuous function $\delta^{\prime}$ the approximately $\delta^{\prime}$-openness of $F$ around $\left(x_{0}, y_{0}\right)$ is equivalent to the following: there 
is $c \in(0,1), r>0$ and $\eta>0$ such that for all $x \in x_{0}+r B_{X}, y \in\left(y_{0}+\eta B_{Y}\right) \cap F(x)$ and $t \in(0, \eta), y+\delta^{\prime}(t) B_{Y} \subset F\left(x+t B_{X}\right)+\delta^{\prime}(c t) B_{Y}$.

Frankowska [12] has introduced the concept of high order variations for multifunctions as follows:

$$
F^{r}\left(x_{0}, y_{0}\right)=\liminf _{(x, y) \stackrel{G r F}{\longrightarrow}\left(x_{0}, y_{0}\right)} \frac{F\left(x+t B_{X}\right)-y}{t^{r}}
$$

where $r>0$ and "lim inf" is taken in the sense of Kuratowski.

In the following corollary we obtain Frankowska's result proved in [13] as a consequence of our main Theorem 1.3 since the $\delta$-regularity implies the $\delta^{\prime}$-openness for some $\delta^{\prime} \leqslant \delta(\operatorname{see}[6,22])$.

Corollary 1.8. Let $X$ and $Y$ be two linear metric spaces, $X$ complete and $r>0$. Assume that

$$
0 \in \text { int } F^{r}\left(x_{0}, y_{0}\right) \text {. }
$$

Then $F$ is $\delta$-regular around $\left(x_{0}, y_{0}\right)$ with $\delta(t)=t^{r}$.

Proof: Since $0 \in$ int $F^{r}\left(x_{0}, y_{0}\right)$ then there is $c>0$ such that for all $0<\varepsilon<c$ there exists $r>0$ satisfying:

$$
t^{r} c B_{Y} \subset F\left(x+t B_{X}\right)-y+\varepsilon t^{r} B_{Y}
$$

for all $x \in x_{0}+r B_{X}, y \in\left(y_{0}+r B_{Y}\right) \cap F(x)$ and $t \in[0, r]$. Put $Z=\mathbb{R}_{+}, G(t)=t B_{X}$, $\delta(t)=t$ and $L(x)=(d(0, x))^{r} c B_{X}$. We easily see that the assumptions of Theorem 1.3 are satisfied.

The following metric regularity result for closed convex multifunctions is an extension of Robinson [24].

Corollary 1.9. Let $X$ and $Y$ be two linear metric spaces with $X$ complete and let $F$ be a multifunction from $X$ into $Y$ with closed convex graph. Then the openness of $F$ at $\left(x_{0}, y_{0}\right) \in G r F$ (that is, there exist $r>0$ such that $y_{0}+r B_{Y} \subset$ $\left.F\left(x_{0}+B_{X}\right)\right)$ is equivalent to the metric $\delta$-regularity of $F$ around $\left(x_{0}, y_{0}\right) \in G r F$, with $\delta(t)=t$.

Proof: In the remainder of the proof we assume for notational convenience that $x_{0}=0$ and $y_{0}=0$; this simply translates the origins in $X$ and $Y$. Suppose that $F$ is open at $(0,0)$. Then by the convexity of $F$ we have for all $0<\varepsilon<r$ and $t \in[0,1]$,

$$
\operatorname{tr} B_{Y} \subset F\left(t B_{X}\right)+\varepsilon t B_{Y} .
$$

So by the convexity of $F$ we have that for all $x \in(r / 2) B_{X}, t \in[0,1 / 2]$ and $y \in$ $(1 / 2) B_{Y} \cap F(x)$,

$$
\operatorname{tr} B_{Y} \subset F\left(x+t B_{X}\right)-y+\varepsilon t B_{Y}
$$


As in Corollary 1.7. we set $Z=\mathbb{R}_{+}, G(t)=t B_{X}, \delta(t)=t$ and $L(x)=d(0, x) r B_{Y}$. Applying Theorem 1.3 we obtain the $\delta$-regularity of $F$ which completes the proof since the other implication is obvious.

Relying on the remark following Theorem 1.3 we obtain a substraction result for convex multifunctions.

Corollary 1.10. If the assumptions of Corollary 1.9 are satisified and if for $0<\alpha<\beta$ one has $y_{0}+\beta B_{Y} \subset F\left(x_{0}+B_{X}\right)+\alpha B_{Y}$, then there is $s>0$ such that,

$$
y_{0}+(\beta-\alpha) s B_{Y} \subset F\left(x_{0}+s B_{X}\right) .
$$

Proof: As in the proof of Corollary 1.9 we asusme that $\left(x_{0}, y_{0}\right)=(0,0)$ and for $r$ sufficiently small one has

$$
t \beta B_{Y} \subset F\left(x+t B_{X}\right)-y+\alpha t B_{Y}
$$

for all $x \in r B_{X}, t \in[0, r]$ and all $y \in r B_{Y} \cap F(x)$. If we set $Z=\mathbb{R}_{+}, G(t)=t B_{X}$ and $L(x)=d(0, x) B_{Y}$, we have $b=1$ and $a=1 / \beta$ where $b$ is as in $H(G)$ and $a$ is the invertibility constant of $L \circ G$. Then it ensues from the remarks following Theorem 1.3 that there is $s>0$ such that for all $y \in y_{0}+(\beta-\alpha) s B_{Y}$ there is $x \in F^{-1}(y)$ satisfying:

$$
d\left(x_{0}, x\right) \leqslant(1 /(\beta-\alpha)) d\left(y_{0}, y\right) \leqslant s
$$

which completes the proof.

\section{REFERENCES}

[1] J.P. Aubin, 'Lipschitz behavior of solutions to convex minimization problems', Math. Oper. Res. 8 (1984), 87-111.

[2] J.P. Aubin and H. Frankowska, 'On the inverse function theorem', J. Math. Pures Appl. 66 (1987), 71-89.

[3] A. Auslender, 'Stability in mathematical programming with nondifferentiable data', SIAM J. Control Optim. (1984), 239-254.

[4] D. Azé, 'An inversion theorem for set-valued maps', Bull. Austral. Math. Soc. 37 (1988), 411-414.

[5] J.M. Borwein, 'Statility and regular point of inequality systems', J. Optim. Theory Appl. 48 (1986), 9-52.

[6] J.M. Borwein and D.M. Zhuang, 'Verifiable necessary and sufficient conditions for openness and regularity for set-valued maps', J. Math. Anal. Appl. 134 (1988), 441-459.

[7] C.C. Chou, 'Inverse function theorems for multifunctions in Frechet spaces' (to appear).

[8] F. Clark, Optimization and nonsmooth analysis (Wiley Interscience, New York, 1983). 
[9] F.S. De Blasi, 'On the differentiability of multifunctions', Pacific J. Math. 66 (1976), 67-81.

[10] S. Dolecki, 'A general theory of necessary optimality conditions', J. Math. Anal. Appl. 7 (1980), 267-308.

[11] I. Ekeland, 'Nonconvex minimization problems', Bull. Amer. Math. Soc. 1 (1979), 443-474.

[12] H. Frankowska, 'An open mapping principle for set-valued maps', J. Math. Anal. Appl. 127 (1987), 172-180.

[13] H. Frankowska, 'Some inverse mapping and open mapping theorems' (to appear).

[14] L.M. Graves, 'Some mapping theorems', Duke Math. J. 17 (1950), 111-114.

[15] J.B. Hiriart-Uruty, 'Refinement of necessary optimality conditions in nondifferentiable programming', Appl. Math. Optim. 5 (1979), 63-82.

[16] A.D. Ioffe, 'Regular points of Lipschitz mapping', Trans. Amer. Math. Soc. 251 (1979), 61-69.

[17] A.D. Ioffe, 'On the local surjection property', Nonlinear Anal. 11 (1987), 565-592.

[18] A. Jourani and L. Thibault, 'Approximate subdifferential and metric regularity: the finite dimensional case', Math. Programming 47 (1990), 203-218.

[19] A. Jourani and L. Thibault, 'The use of metric graphical regularity in approximate subdifferential calculus rules in finite dimensions', Optimization 21 (1990), 509-519.

[20] L.V. Kantorovich and G.P. Akilov, Functional analysis in normed spaces (Macmillan, New York, 1964).

[21] J.P. Penot, 'On regularity conditions in mathematical programming', Math. Programming Stud. 19 (1982), 167-199.

[22] J.P. Penot, 'Metric regularity, openness and Lipschitz behavior of multifunctions', Nonlinear Anal. 13 (1989), 629-643.

[23] S.M. Robinson, 'Stability theorems of systems of inequality part II: Differentiable nonlinear systems', SIAM J. Numer. Anal. 13 (1976), 497-513.

[24] S.M. Robinson, 'Regularity and stability for convex multivalued functions', Math. Oper. Res. 1 (1976), 130-143.

[25] R.T. Rockafellar, 'Lipschitzian properties of multifunctions', Nonlinear Anal. 9 (1985), 867-885.

[26] R.T. Rockafellar, 'Extensions of subgradient calculus with application to optimization', Nonlinear Anal. 9 (1985), 665-698.

[27] L. Thibault, 'On subdifferential of optimal value function', SIAM J. Control Optim. (to appear).

[28] J. Zowe and S. Kurcyusz, 'Regularity and stability for mathematical programming problems in Banach spaces', Appl. Math. Optim. 5 (1979), 49-62.

Mathématiques

Université de Perpignan

66025 Perpignan Cedex

France 